\title{
Avaliação e comparação entre métodos de mensuração de pressão arterial sistólica em gatos hígidos anestesiados
}

[Evaluation and comparison between methods of measurement of systolic blood pressure in healthy anesthetized cats]

\author{
T.M. Anjos ${ }^{1}$, J.C.C. Veado $^{1}$, M.C.N. Castro $^{2}$, S.A. Diniz ${ }^{1}$, G.S.L. Rocha ${ }^{3}$, E.F. Silva ${ }^{3}$, C.A.V. Araújo ${ }^{3}$, \\ C.D. Freitas ${ }^{3}$, M.Q. Maia ${ }^{3}$, C.A.P. Tavares ${ }^{3}$
}

${ }^{1}$ Escola de Veterinária - Universidade Federal de Minas Gerais - UFMG - Belo Horizonte, MG

${ }^{2}$ Faculdade de Veterinária - Universidade Federal Fluminense - UFF - Niterói, RJ

${ }^{3}$ Médico veterinário autônomo

\begin{abstract}
RESUMO
Foram avaliados e comparados, para fins de validação, dois diferentes métodos de mensuração de pressão arterial sistólica (PAS), não invasivos ou indiretos (oscilométrico e Doppler vascular) com o invasivo ou direto (cateterização arterial - padrão ouro), em 12 gatos hígidos anestesiados de idade variando entre sete meses e dois anos. Em cada animal, procedeu-se à mensuração simultânea da pressão não invasiva e da invasiva. O método oscilométrico foi comparado, por meio de sete medidas consecutivas, com intervalo de 30 segundos entre elas, com o método invasivo, e, logo em seguida, o método Doppler foi comparado ao método invasivo da mesma maneira. Concluiu-se que o método oscilométrico é estatisticamente igual ao método invasivo, enquanto o Doppler difere de ambos. Entretanto, pela forte correlação positiva entre os métodos Doppler e invasivo, foi possível criar um fator de correção (equação de regressão linear) para se determinar o valor da pressão invasiva, a partir do valor obtido pelo método Doppler. Para obtenção de valores mais fidedignos, recomenda-se a utilização desse fator de correção quando o método Doppler for utilizado para mensuração de PAS em gatos anestesiados.
\end{abstract}

Palavras-chave: felino, doppler, pressão arterial

\begin{abstract}
For validation purposes, two different methods of measurement of systolic blood pressure (SBP), noninvasive or indirect (oscillometric and vascular doppler), direct or invasive (arterial catheterization - the gold standard) were evaluated and compared in 12 healthy anesthetized cats, varying between seven months to two years old. In each animal, we proceeded to the simultaneous noninvasive and invasive measurement of pressure. The oscillometric method was compared by means of seven consecutive measurements with an interval of 30 seconds between them, with the invasive method, and soon after, the doppler method was compared to the invasive method in the same way. It was concluded that the oscillometric method is statistically equal to the invasive method, whereas doppler differs from both. However, due to the strong positive correlation between doppler and invasive methods, it was possible to create a correction factor (linear regression) to determine the value of invasive pressure from the value obtained by the doppler method. To obtain more reliable figures, it is recommended to use this correction factor when the doppler method is used to measure SBP in anesthetized cats.
\end{abstract}

Keywords: feline, doppler, blood pressure

\section{INTRODUÇÃO}

A ocorrência de alterações pressóricas é um achado comum e frequente em pequenos animais. A hipotensão, decorrente, por exemplo,

Recebido em 3 de abril de 2013

Aceito em 17 de dezembro de 2013

E-mail: tathimouraoanjos@hotmail.com de procedimentos anestésicos, e a hipertensão arterial sistêmica, secundária principalmente à doença renal crônica e ao hipertireoidismo, são bastante prevalentes na espécie felina (Brown $e t$ al., 2007). Por isso, merecem avaliação e acompanhamento constante na rotina clínica, 
permitindo, dessa maneira, a instituição de manejo e terapia adequados a cada caso. Assim, a mensuração da pressão arterial (PA) tem sido considerada de grande importância e vem ocupando lugar de destaque na medicina veterinária (Henik et al., 2005), principalmente em consequência da maior disponibilidade de aparelhos indiretos (não invasivos) de mensuração de PA.

A PA pode ser avaliada tanto por métodos indiretos quanto por método direto. $\mathrm{O}$ método direto ou invasivo oferece vantagens, como monitoramento contínuo e preciso da PA, mesmo em condições de hipotensão grave, como em condições de hipertensão, sendo a mensuração intra-arterial considerada o padrão ouro (Brown et al., 2007). Em gatos, a cateterização intraarterial pode ser realizada por meio de dissecção de uma artéria ou de forma percutânea, sendo mais facilmente realizada na artéria femoral, embora a artéria metatársica dorsal também possa ser utilizada (Trim, 1994). Raramente a técnica invasiva é utilizada na rotina devido às dificuldades e aos riscos, que incluem sedação e/ou anestesia (Rezende et al., 2002; Henik et al., 2005; Acierno et al., 2010), fatores que, além de pouco práticos, podem reduzir artificialmente a PA. Além disso, é de difícil execução em animais de peso abaixo de $10 \mathrm{~kg}$, sendo desaconselhado seu uso em gatos (Stepien e Elliott, 2007). Complicações comuns decorrentes da cateterização são isquemia, formação de trombos, além de embolia. Por tais motivos, o método invasivo é mais utilizado em pesquisas acadêmico-científicas e unidades de terapia intensiva.

Os métodos indiretos ou não invasivos, como o Doppler e o oscilométrico, são preferencialmente utilizados em situações clínicas, devido à maior praticidade de uso e à possibilidade de ser repetido em pequenos intervalos de tempo (Podell, 1992). Entretanto, são menos precisos em condições de hipotensão, vasoconstrição ou ainda quando ocorre movimentação excessiva do animal, podendo subestimar o verdadeiro valor da PA (Podell, 1992; Henik et al., 2005).

Atualmente, tem-se verificado um número crescente de aparelhos indiretos no mercado. Embora tenham seu uso já consagrado, os métodos indiretos de mensuração de PA ainda deixam dúvidas quanto à sua acurácia e praticidade na rotina clínica (Acierno et al., 2010), sendo necessário comparar e estabelecer métodos e aparelhos confiáveis que verdadeiramente auxiliem na mensuração fidedigna da PA de gatos. Além disso, apesar de fabricados em série e testados pelo fabricante, faz-se necessário validá-los, já que a adequação às normas é o que garante sua confiabilidade (Furusawa, 2005).

A melhor forma de se avaliar e validar um método indireto é compará-lo, de modo pareado, ao método invasivo (Bland e Altman, 1986). O Instituto Americano Nacional de Padrões (ANSI) reconhece a recomendação publicada na normativa de 2001 do Comitê Europeu de Organização (CEN), que valida o método indireto se a diferença da pressão arterial diastólica (PAD) comparada ao método invasivo não for maior que $8 \mathrm{mmHg}$ e/ou se a PAS não for maior que $12 \mathrm{mmHg}$ (Association for the Advancement of Medical Instrumentation, 2003). De acordo com Brown et al. (2007), nenhum aparelho de mensuração indireta de PA ainda foi validado em medicina veterinária pelos critérios ora mencionados.

Objetivou-se com este estudo correlacionar os valores médios de PAS obtidos por dois métodos indiretos com os valores obtidos pelo método direto (cateterização arterial), em um grupo de gatos hígidos, de idade variando entre sete meses e dois anos, durante anestesia geral, validando, dessa maneira, os métodos indiretos para uso na rotina clínica.

\section{MATERIAL E MÉTODOS}

Foram selecionados 12 gatos (Felis catus) hígidos, sendo seis machos e seis fêmeas, com idade compreendida entre sete meses e dois anos de idade, devidamente vacinados e desparasitados, mestiços, não castrados, de peso compreendido entre 2,88 e $3,94 \mathrm{~kg}$. Todos os gatos foram agrupados em um mesmo grupo denominado Junior - segundo classificação etária proposta pelas "Diretrizes de estágio de vida felina" estabelecidas pela AAFP-AAHA (American Association of Feline Practioners American Animals Hospital Association) (Vogt et al., 2010). Todos os gatos selecionados foram submetidos aos exames: físico completo (incluindo fundoscopia ocular), laboratoriais (sangue - FIV/FeLV, hemograma completo, 
proteína total e frações, bioquímica renal, hepática e hormonal, além de urina - urinálise e relação proteína:creatinina urinária) e de imagem (ultrassom abdominal e ecodopplercardiograma) para comprovação de higidez.

Após jejum alimentar de oito horas e hídrico de duas horas, os gatos foram anestesiados. A medicação pré-anestésica foi realizada com acepromazina (Acepran 0,2\%, Vetnil, Brasil), na dose de $0,03 \mathrm{mg} / \mathrm{kg}$, associada à meperidina (Petinan 50mg/mL, BioChimico, Brasil), na dose de $3 \mathrm{mg} / \mathrm{kg}$, na mesma seringa, por via intramuscular. Após tranquilização adequada, tricotomia e assepsia prévia, um cateter n.24 intravenoso foi introduzido no membro torácico esquerdo e conectado a um equipo microgotas, para fluidoterapia com solução de ringer com lactato mantida por bomba de infusão volumétrica peristáltica. Foi administrado, por via endovenosa, o agente indutor propofol $1 \%$ (Provive $1 \%$, Claris, Índia), na dose de $5 \mathrm{mg} / \mathrm{kg}$. Para evitar intubação traumática e prevenir ocorrência de laringoespasmo, a região laríngea foi previamente dessensibilizada com aspersão de lidocaína 2\% (Lidovet 2\%, Bravet, Brasil). Após perda do reflexo laríngeo, com auxílio de laringoscópio, foi realizada intubação orotraqueal, com tubo endotraqueal de calibre adequado. O tubo foi conectado ao monitor de apneia, e este conectado ao aparelho de anestesia inalatória (sistema circular semifechado) para permitir a inalação do anestésico volátil isoflurano (Isoforine, Cristália, Brasil), na concentração de $1,5 \%$, pelo gato. Colírio lubrificante era instilado nos dois olhos periodicamente para evitar ressecamento corneal.

Após a obtenção de plano anestésico, cada gato foi colocado em decúbito dorsal sob colchão térmico para evitar perda de temperatura corporal. A pressão arterial média (PAM) invasiva foi mantida entre 70 e $100 \mathrm{mmHg}$, a frequência cardíaca entre 100 e 200 bpm e a frequência respiratória entre 10 e $20 \mathrm{mpm}$, por meio de alteração na velocidade da bomba de infusão de fluidoterapia, concentração do anestésico inalatório e ventilação controlada. O monitoramento dos parâmetros vitais foi realizado por monitor multiparâmetro veterinário (Life Window Lite LW8 Vet, Digicare Animal Health, Estados Unidos), por meio de traçado eletrocardiográfico, oximetria de pulso, frequência cardíaca, frequência respiratória, fração inspirada de oxigênio e temperatura corporal.

Foram realizadas sete mensurações invasivas e não invasivas, pareadas e simultâneas, com intervalo de 30 segundos entre elas. Inicialmente, o método oscilométrico (membro torácico direito) foi comparado com o método invasivo (membro pélvico direito - artéria femoral), e, cinco minutos após o término, o mesmo procedimento foi repetido, entretanto empregando-se o método Doppler (membro torácico direito). Para os métodos não invasivos, utilizou-se o mesmo manguito, conforme recomendações do fabricante. Para fins de validação, utilizaram-se os parâmetros determinados pela normativa de 2001 do CEN (Comitê Europeu de Organização).

Para obtenção de valores confiáveis de PA, todas as mensurações foram realizadas somente por um único examinador bem treinado, com experiência em mensuração de PA e familiaridade com os métodos e aparelhos estudados há mais de três anos. A primeira medida de PA obtida por todos os métodos e as demais obtidas com valores extremos (variação>20\% PAS) foram excluídas. Foi seguido o protocolo (Brown et al., 2007) elaborado pelo Consenso "Diretrizes para a Identificação, Avaliação e Manejo de Hipertensão Arterial Sistêmica em Cães e Gatos" criado pelo Colégio Americano de Medicina Veterinária Interna e pela Sociedade Veterinária de Pressão Arterial.

Após tricotomia e assepsia prévia do membro pélvico direito, a artéria femoral foi dissecada e nela introduziu-se um cateter n.24 (Angiocath, 24G, BD, Estados Unidos). Este foi acoplado a um transdutor elétrico de pressão (Transdutor de Pressão II, Utah Medical Products Inc., Estados Unidos) conectado ao monitor multiparâmetro por meio de sistema (Sistema de fluxo Deltran®, Utah Medical Products Inc., Estados Unidos) composto por tubo extensor e torneira de três vias, posicionado entre a extensão e o transdutor.

Para mensuração precisa, o transdutor de pressão estava posicionado à altura do coração do paciente. Fechou-se o transdutor para o paciente e abriu-se para o ambiente, momento no qual os valores do monitor multiparâmetro foram zerados. Em seguida, o transdutor foi fechado 
para o ambiente, e a comunicação aberta para o paciente, iniciando-se os registros da PA invasiva. Visualizaram-se, na tela do monitor multiparâmetro, os valores digitais de pressão arterial sistólica, pressão arterial média, pressão arterial diastólica, frequência cardíaca e os demais parâmetros biológicos de monitoramento já mencionados.

Todo o sistema compreendido entre paciente e transdutor foi mantido preenchido com solução de cloreto de sódio $0,9 \%$ heparinizada $(0,2 \mathrm{~mL}$ de heparina 5.000UI/mL em $100 \mathrm{~mL}$ de $\mathrm{NaCl} 0,9 \%$ ) para evitar coagulação do sangue e trombose, bem como foi mantido isento de bolhas de ar para não haver diminuição na amplitude do pulso, com consequente interferência nos valores de pressão obtidos (Trim, 1994).

Para mensuração pelo método oscilométrico (Petmap®, Ramsey Medical, Inc., Estados Unidos), a escolha do manguito (CritterCuff size - $3.0 \mathrm{~cm} / 3.5 \mathrm{~cm} / 4.0 \mathrm{~cm}$, Ramsey Medical, Inc., Estados Unidos) era realizada conforme recomendações do fabricante, e o manguito era colocado sempre no membro torácico direito sobre o terço distal da região radioulnar.

O membro foi mantido posicionado no nível do coração direito. O manguito, então, foi automaticamente inflado com um aperto contínuo da pera ao longo de um a três segundos a uma pressão suprassistólica $(40-50 \mathrm{mmHg})$ e, em seguida, desinflado automaticamente de modo progressivo. As oscilações da parede arterial emitidas pela pulsação sanguínea eram captadas pelo manguito e permitiam ao aparelho oscilométrico determinar a oscilação máxima e, consequentemente, calcular a PAM por meio do microprocessador contido dentro do aparelho. Logo, por cálculo aritmético, o aparelho determinava as PAS, PAD, além da frequência cardíaca. Para que nova mensuração pudesse ser realizada sem interferência, o manguito era totalmente desinflado, a fim de normalizar mais rapidamente a estase venosa (Henik et al., 2005).

Após cinco minutos do término da técnica oscilométrica, o animal era mantido na mesma posição e com o mesmo manguito, para mensuração pelo Doppler vascular (Model $812^{\circledR}$,
Parks Medical, Estados Unidos). O manguito era, então, desconectado do aparelho oscilométrico e conectado ao esfigmomanômetro aneroide (Esfigmomanômetro Gamma G5 ${ }^{\circledR}$, Heine, Alemanha). Álcool $70^{\circ}$ e gel de ecografia foram aplicados sobre a superfície do transdutor (Flat infant probe 8,2MHz, Parks Medical Electronics, Inc., Estados Unidos) e sobre o pelo da região palmar metacarpal (artéria digital palmar comum) próxima ao coxim, onde o pulso era audível.

Após obtenção dos sinais de pulso adequados, o manguito foi inflado até aproximadamente 40 a $50 \mathrm{mmHg}$ superior à pressão necessária para se obliterar o sinal de pulso audível e, logo após, a válvula do esfigmomanômetro foi aberta lentamente, na velocidade de 2 a $5 \mathrm{mmHg}$ por segundo, para que o manguito desinflasse lentamente. A PAS foi determinada no momento em que o sinal de pulso se tornou audível (claro e consistente) novamente, e a PAD foi desconsiderada por ser subjetiva quando obtida pelo Doppler (Henik et al., 2005).

Após o término das mensurações, foi removido o cateter da artéria femoral e realizou-se compressão manual sobre o local por cinco minutos. A pele, então, foi suturada (Nylon 3-0, Ethicon, Estados Unidos) com pontos simples separados, e nova compressão manual foi realizada, por 15 a 20 minutos, para minimizar a ocorrência de hemorragia.

Por se tratarem de animais de tutores, não foram submetidos a protocolos que induzissem hipotensão e/ou hipertensão, para fins de comparação entre os métodos. O projeto foi aprovado pelo Comitê de Ética em Experimentação Animal (CETEA/UFMG), sob o protocolo de número 223/2011, com validade até 19/10/2016.

Os dados obtidos foram analisados pela análise de variância (ANOVA), com teste pós-ANOVA, utilizando-se teste " $t$ " de Student $(\mathrm{P}<0,05)$ para blocos ao acaso, sendo os animais considerados os blocos. As análises foram feitas com auxílio dos programas computacionais (softwares) BioStat 2009 Professional 5.8.4 e Microsoft Excel. 


\section{RESULTADOS E DISCUSSÃO}

Na Tab. 1, apresentam-se os valores médios, os desvios-padrão, as diferenças e o coeficiente de correlação (r) de PAS obtidos pelos métodos invasivo e não invasivo.

Na Fig. 1, observa-se que a média dos valores obtidos pelo método Doppler vascular foi significativamente menor que as médias obtidas tanto pelo método invasivo (diferença de $12,58 \mathrm{mmHg}$ - 12,22\%) quanto pelo método oscilométrico (diferença de 12, 27mmHg $11,96 \%)$. Provavelmente, essa subestimação de valores obtidos pelo Doppler foi observada em decorrência do protocolo anestésico realizado neste estudo, já que os gatos apresentaram valores de PAS mais baixos, justificando a limitação desse método em condições de hipotensão. Entretanto, não houve diferença significativa entre a média dos valores obtidos pelo método oscilométrico e a média dos valores obtidos pelo método invasivo (diferença de $1,25 \mathrm{mmHg}-1,20 \%)$.

Tabela 1. Valores médios, desvios-padrão, diferenças (em mmHg e porcentagem) de PAS obtidos pelos métodos invasivo e não invasivo (oscilométrico e Doppler)

\begin{tabular}{cccc}
$\begin{array}{c}\text { Invasivo (mmHg) } \\
\text { (Média / Desvio-padrão) } \\
\text { (Mínimo - Máximo) }\end{array}$ & $\begin{array}{c}\text { Oscilométrico (mmHg) } \\
\text { (Média / Desvio-padrão) } \\
\text { (Mínimo - Máximo) }\end{array}$ & $\begin{array}{c}\text { Diferença entre as médias } \\
\text { Métodos não invasivo e } \\
\text { invasivo (mmHg e \%) }\end{array}$ & $\begin{array}{c}\text { Coeficiente } \\
\text { de correlação } \\
\text { (r) }\end{array}$ \\
\hline $\begin{array}{c}103,84 \pm 17,01^{\mathrm{a}} \\
(87,29-135,86)\end{array}$ & $\begin{array}{c}102,59 \pm 16,69^{\mathrm{a}} \\
(82,57-135,71)\end{array}$ & $1,25 \mathrm{mmHg} / 1,20 \%$ & 0,9740 \\
\hline $\begin{array}{c}\text { Invasivo }(\mathrm{mmHg}) \\
\text { (Média / Desvio-padrão) } \\
(\text { Mínimo - Máximo) }\end{array}$ & $\begin{array}{c}\text { Doppler }(\mathrm{mmHg}) \\
\text { (Média/Desvio-padrão) } \\
\text { (Mínimo - Máximo) }\end{array}$ & $\begin{array}{c}\text { Diferença entre as médias } \\
\text { Métodos não invasivo e } \\
\text { invasivo (mmHg e \%) }\end{array}$ & $\begin{array}{c}\text { Coeficiente } \\
\text { de correlação } \\
\text { (r) }\end{array}$ \\
\hline $\begin{array}{c}102,90 \pm 17,02^{\mathrm{a}} \\
(82,14-137,29)\end{array}$ & $\begin{array}{c}90,32 \pm 17,49^{\mathbf{b}} \\
(61,43-119,29)\end{array}$ & $12,58 \mathrm{mmHg} / 12,22 \%$ & 0,9266
\end{tabular}

Letras iguais na mesma linha indicam igualdade estatística, e letras distintas na mesma linha indicam diferença estatística pelo teste " $t$ " de Student para $\mathrm{P}<0,05$.

A diferença média (bias ou viés) foi calculada (Tab. 1) para cada série de mensurações, subtraindo-se o valor médio de PAS obtido pelo método invasivo pelo método não invasivo. Um viés positivo indica que a medida indireta (não invasiva) subestima a medida direta (invasiva padrão ouro), e um viés negativo indica que a medida indireta superestima a medida correspondente direta (Bosiack et al., 2010).

Os métodos Doppler e invasivo, apesar de terem médias significativamente distintas, apresentaram correlação alta e positiva $(\mathrm{r}=$ $0,9266, \mathrm{R}^{2}=0,8586$ e $\mathrm{P}<0,05$ ) (Fig. 2). Foi possível, por regressão linear, criar uma equação: $y=0,9 x+21,5$, em que $x$ representa um valor de PAS obtido pelo Doppler e $y$ representa o valor calculado que estima a PAS que seria obtida pelo método invasivo. Já que o método oscilométrico apresentou forte correlação positiva $(r=0,9740$, $\mathrm{R}^{2}=0,9486$ e $\left.\mathrm{P}<0,05\right)$ e não diferiu significativamente do método invasivo, não foi necessária a criação de uma equação.
Por ser menos sensível ao padrão respiratório e à movimentação do paciente, apresentar valores consistentes, ter um menor tempo de operação para obtenção de resultados, além do baixo custo, quando comparado ao método oscilométrico, o Doppler é o método de mensuração de PA mais empregado em medicina felina (Brown et al., 2007). Entretanto, no presente estudo, observou-se, que, apesar da forte correlação $\left(\mathrm{r}=0,9266, \mathrm{R}^{2}=0,8586 \mathrm{e}\right.$ $\mathrm{P}<0,05)$ com o método invasivo, o método Doppler tende a subestimar a PAS em $12,58 \mathrm{mmHg}$ (diferença de 12,22\%). Essa subestimação também foi observada em outros trabalhos (Grandy et al., 1992; Binns et al., 1995; Caulkett et al., 1998; Pedersen, 2002). É sabido que, em casos de hipotensão, o Doppler é menos preciso e subestima os valores de PA (Stepien e Elliott, 2007), e possivelmente, em decorrência do protocolo anestésico realizado no presente estudo, os gatos apresentaram valores de PAS mais baixos, o que justificaria a limitação desse método. 


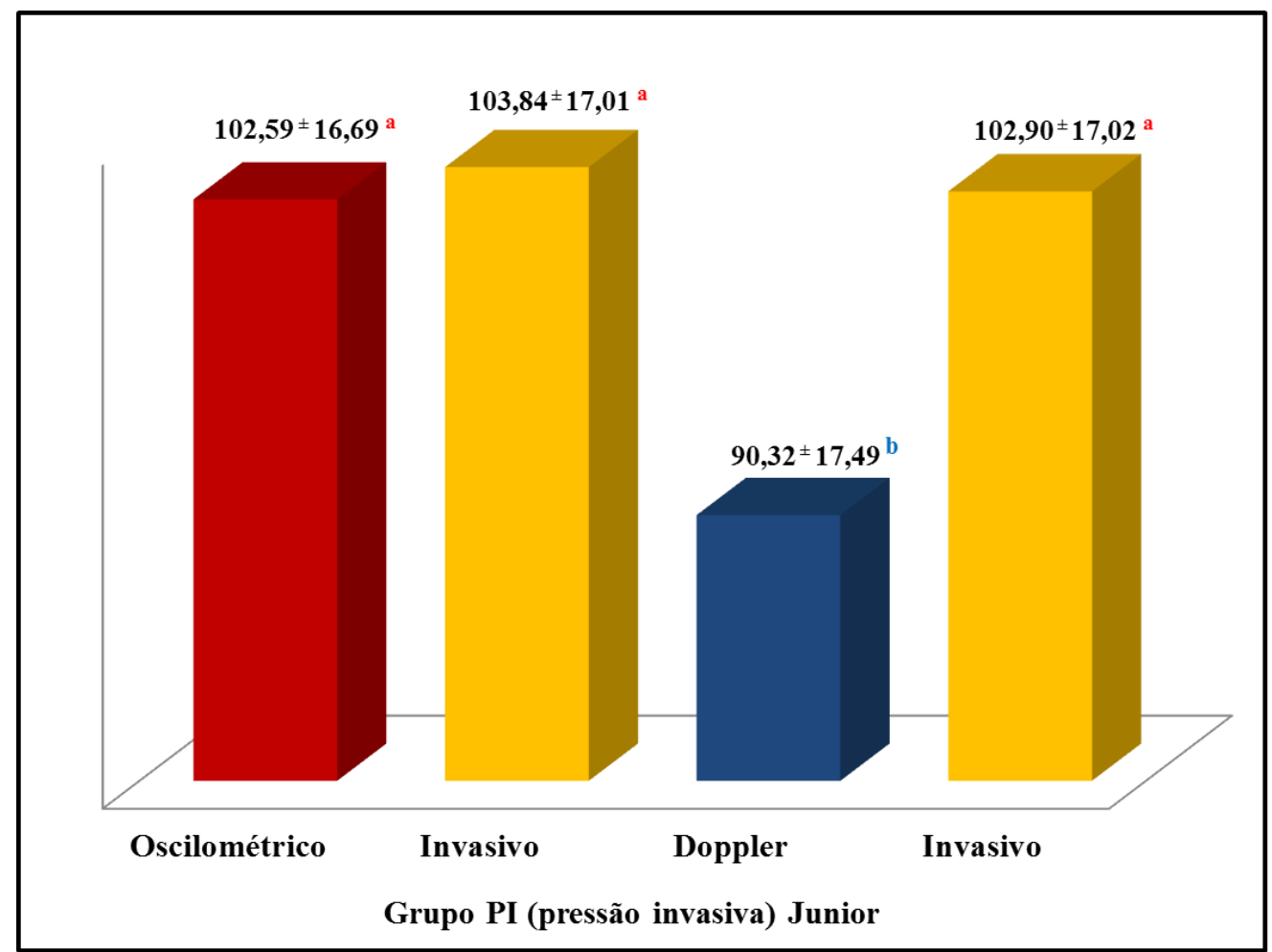

Figura 1. Representação gráfica dos valores médios e desvios-padrão (mmHg) de PAS obtidos pelos métodos invasivo e não invasivo. Letras distintas indicam diferença estatística e letras iguais indicam igualdade estatística pelo teste " $\mathrm{t}$ " de Student para $\mathrm{P}<0,05$.

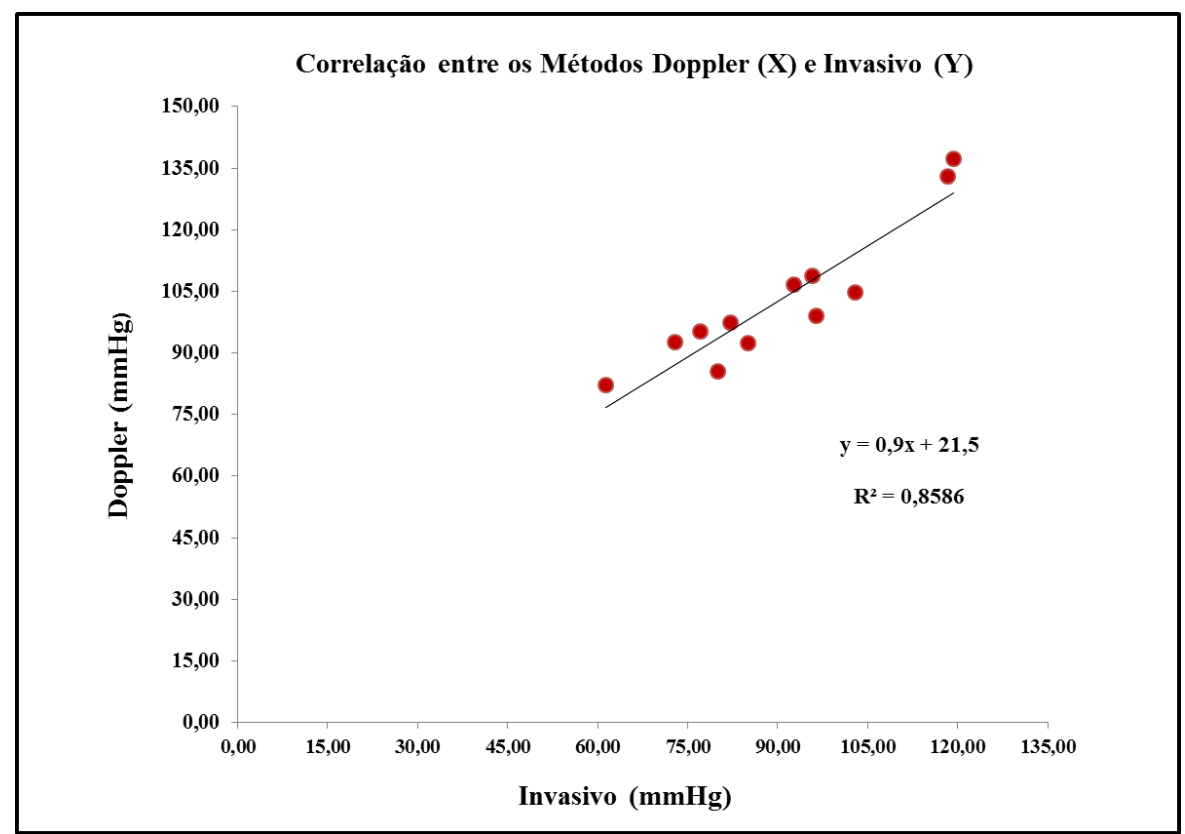

Figura 2. Correlação gráfica entre os métodos Doppler e invasivo por regressão linear simples $(\mathrm{P}<0,05)$. 
Alguns autores (Brown e Henik, 1998; Acierno e Labato, 2004) comentam que o método oscilométrico não deve ser utilizado para gatos ou cães pequenos (com menos de $10 \mathrm{~kg}$ ) porque essa técnica subestima a PA desses animais. Entretanto, o aparelho oscilométrico, testado neste estudo (PetMap ${ }^{\circledR}$, Ramsey Inc.), apresentou igualdade estatística e alta correlação ( $\mathrm{r}=$ $0,9740, \quad \mathrm{R}^{2}=0,9486$ e $\left.\mathrm{P}<0,05\right)$ quando comparado ao método invasivo (diferença de $1,25 \mathrm{mmHg}-1,20 \%$ ), provavelmente devido ao seu sistema algoritmo otimizado (variação de \pm 2 $\mathrm{mmHg}$ ) pelo seu microprocessador interno e específico, que permite que seja selecionada a espécie (canina ou felina) em que será realizada a mensuração da PA, bem como o local de colocação do manguito (membro torácico, cauda ou membro pélvico - ordem de preferência do fabricante). Dessa maneira, a otimização do aparelho (de acordo com a melhor correlação obtida com a pressão arterial invasiva, de cada espécie estudada, no momento em que foi desenvolvido) permite a obtenção de valores fidedignos.

No presente estudo, optou-se por trabalhar conforme as recomendações expressas no manual de instruções (selecionado para espécie felina e para o membro torácico - melhor local de mensuração segundo o fabricante). De acordo com o manual do aparelho oscilométrico em questão, o resultado dessa otimização é que as medidas de PA obtidas por esse aparelho serão em torno de 10 a $20 \%$ mais elevadas que as medidas obtidas pelos demais métodos indiretos oscilométrico (outros aparelhos) e Doppler, e que a diferença entre entre esse aparelho e a pressão invasiva será de $\pm 2 \mathrm{mmHg}$.

Ainda segundo o fabricante, a melhor precisão nas medidas de PA obtidas é alcançada utilizando-se o manguito de tamanho certo, com as configurações adequadas para as espécies e o local de colocação do manguito, na função de média que é oferecida pelo aparelho (após as mensurações seriadas, desliga-se o aparelho e liga-se novamente; na tela aparecerá a leitura mais representativa daquela série de mensurações realizadas).

Os resultados obtidos neste estudo diferem dos resultados obtidos por Acierno et al. (2010), que compararam três aparelhos oscilométricos (Petmap ${ }^{\circledR}$ e outros dois aparelhos) com o método invasivo em gatos anestesiados. O Petmap ${ }^{\circledR}$, entretanto, subestimou a PAS invasiva em $14,9 \mathrm{mmHg}$. Não foi informado se ele foi utilizado no modo otimizado (espécie felina), conforme recomendado. O método invasivo foi realizado na artéria podal dorsal, diferente do presente estudo, em que foi realizado na artéria femoral. O protocolo anestésico também diferiu do protocolo do presente estudo. Apesar de a quetamina ter sido associada ao midalozam, que, em teoria, anularia seus efeitos cardiovasculares (taquicardia), não se pode afirmar que a frequência cardíaca não estivesse mais elevada, tornando, assim, a mensuração oscilométrica imprecisa.

O valor de PAS obtido pelo método oscilométrico foi $11,96 \%$ superior ao valor obtido pelo método Doppler, estando dentro dos valores referidos pelo fabricante. Quando comparado ao método invasivo, o método oscilométrico apresentou uma diferença menor que $2 \mathrm{mmHg}(1,25 \mathrm{mmHg})$, conforme referido pelo fabricante. Durante a mensuração simultânea da PAS pelos métodos invasivo e oscilométrico, não houve diferença significativa entre os valores de frequência cardíaca detectada por ambos os aparelhos, atestando a confiabilidade do método indireto oscilométrico estudado. Para permitir uma melhor correlação com a medida obtida de maneira invasiva (cateterização intra-arterial), houve uma preocupação, neste estudo, quanto à metodologia para a realização da presente pesquisa no que se refere à espécie estudada e à localização de colocação do manguito. Desse modo, mensurouse a PA pelo método oscilométrico no modo otimizado para a espécie felina e para o manguito colocado no membro torácico.

No estudo em questão, os métodos indiretos, comparados ao método direto, poderiam ser considerados como validados de acordo com a normativa de 2001 do CEN (que valida quando a diferença média de PAS entre os métodos não é maior que $12 \mathrm{mmHg}$ ).

As complicações ocorridas em decorrência da cateterização da artéria femoral foram: hemorragia profusa, hematoma, inchaço de membro, formação de coágulo, e um animal apresentou tromboembolismo arterial com perda da propriocepção, tônus, temperatura e pulso no membro. Essas complicações são descritas por 
Jones (1996), e, segundo esse autor, a isquemia, a trombose e a embolia são as mais frequentes. A isquemia ocorre pela formação de um trombo em um vaso ou embolização de material particulado ou ainda coágulos que se formam no leito vascular, ou são introduzidos durante as manobras de irrigação do sistema. A trombose ocorre pela presença do cateter no vaso, lesando o endotélio ou obstruindo mecanicamente a luz arterial. Pode ainda estar relacionada à estase sanguínea, como na hipotensão arterial (baixo débito cardíaco) ou após pressão excessiva e prolongada sobre o local da punção, após a retirada do cateter.

Todos se recuperaram, inclusive a gata acometida por tromboembolismo, após 15 dias de terapia medicamentosa: heparina sódica (Hemofol 5000UI/mL 10mL, Cristália, Brasil) 200UI/kg/SC/TID a cada $72 \mathrm{~h}+$ aspirina (Aspirina Prevent 100mg Enteric coated, Bayer Schering Pharma, Alemanha) - 10mg/kg/VO, 2 x semana + rutosídeo 500mg (Venoruton rutosídeo 500mg, Novartis, Suiça) - 50mg/kg/BID associada a 30 dias de fisioterapia, compressa quente e eletroestimulação diária do membro.

\section{CONCLUSÃO}

Os métodos indiretos (Doppler e oscilométrico) estudados, quando comparados ao método direto (padrão ouro), podem ser considerados validados para felinos hígidos anestesiados, independentemente do sexo. Entretanto, recomenda-se o uso do fator de correção quando o método Doppler for utilizado para mensuração de PAS em gatos anestesiados nas mesmas condições e idade em que foram realizadas neste estudo, para obtenção de valores mais fidedignos.

\section{REFERÊNCIAS}

ACIERNO, M.J.; LABATO, M.A. Hypertension in dogs and cats. Compendium on Cont. Educ. for Pract. Vet., v.26, p.336-345, 2004.

ACIERNO, M.J.; SEATON, D.; MITCHELL, M.A. et al. Agreement between directly measured blood pressure and pressures obtained with three veterinary-specific oscillometric units in cats. J. Am. Vet. Med. Associat., v.237, p.402406, 2010.
ASSOCIATION for the Advancement of Medical Instrumentation. Manual, electronic, or automated sphygmomanometers. 2003. Arlington: Association for the advancement of medical instrumentation. $586 \mathrm{p}$.

BINNS, S.H.; SISSON, D.D.; BUOSCIO, D.A. et al. Doppler Ultrasonographic, Oscilometric Sphygmomanometric, and Photoplethysmografic Techniques for Noninvasive Blood Pressure Measurement in Anesthetized Cats. J. Vet. Internal Med., v.9, p.405-414, 1995.

BLAND, J.M.; ALTMAN, D.G. Statistical Methods for Assessing Agreement between Two Methods of Clinical Measurement. The Lancet, v.327, p.307-310, 1986.

BOSIACK, A.P.; MANN, F.A.; DODAM, J.R. et al. Comparison of ultrasonic Doppler flow monitor, oscillometric, and direct arterial blood pressure measurements in ill dogs. J. Vet. Emerg. Critical Care, v.20, p.207-215, 2010.

BROWN, S.; ATKINS, C.; BAGLEY, R. et al. Guidelines for the identification, evaluation, and management of systemic hypertension in dogs and cats. J. Vet. Internal Med., v.21, p.542-558, 2007.

BROWN, S.A.; HENIK, R.A. Diagnosis and treatment of systemic hypertension. Vet. Clin. North Am.: Small Anim. Pract., v.28, p.14811494, 1998.

CAULKETT, N.A.; CANTWELL, S.L.; HOUSTON, D.M. A comparison of indirect blood pressure monitoring techniques in the anesthetized cat. Vet. Surg., v.27, p.370-377, 1998.

FURUSAWA, E.A.; RUIZ, O.; SAITO, M.I. et al. Avaliação do monitor de medida de pressão arterial Omron 705-CP para uso em adolescentes e adultos jovens. Arq. Bras. Cardiol., v.84, p.367-370, 2005.

GRANDY, J.L.; DUNLOP, C.I.; HODGSON, D.S. et al. Evaluation of the Doppler ultrasonic method of measuring systolic arterial blood pressure in cats. Am. J. Vet. Res., v.53, p.11661169, 1992.

HENIK, R.A; DOLSON, M.K.; WENHOLZ, B.S. How to obtain a blood pressure measurement. Clin. Techniques in Small Anim. Pract., v.20, p.144-150, 2005. 
JONES, J.L. Invasive monitoring techniques in anesthesized animals. Vet. Med., v.91, p.337340, 1996.

PEDERSEN, K.M.; BUTLER, M.A.; ERSBOLL, A.K. et al. Evaluation of an oscillometric blood pressure monitor for use in anesthetized cats. $J$. Am. Vet. Med. Associat., v.221, p.646-650, 2002.

PODELL M. Use of blood pressure monitors. In: BONAGURA, J.D. Kirk's Current Veterinary Therapy XI: Small Animal Practice. Philadelphia, PA: WB Saunders, 1992. p.834837.

REZENDE, M.L.; NUNES, N.; SOUZA, A.P.; SANTOS, P.S.P. Monitoramento hemodinâmico invasivo em pequenos animais. Semina: Cienc. Agr., v.23, p.93-100, 2002.
STEPIEN, R.L.; ELLIOTT, J. Measurement of blood pressure. In: ELLIOTT, J.; GRAUER, G.F. BSAVA Manual of Canine and Feline Nephrology and Urology. UK: British Small Animal Veterinary Association, 2007. p.178-191.

TRIM, C.M. Monitoring the anaesthetized cat. In: HALL, L.W.; TAYLOR, P.M. Anaesthesia of the cat. London: Baillière Tindal, 1994. p.194223.

VOGT, A.H.; RODAN, I.; BROWN, M. et al. AAFP - AAHA feline life stage guidelines. $J$. Feline Med. Surg., v.12, p.43-54, 2010. 